\title{
Stable and destabilized GFP reporters to monitor calcineurin activity in Saccharomyces cerevisiae
}

\author{
Jutta Diess $\left.\right|^{1}$, Arpita Nandy ${ }^{1}$, Christina Schug ${ }^{1}$, Lukas Habernig ${ }^{1}$ and Sabrina Büttner ${ }^{1,2, *}$ \\ 1 Department of Molecular Biosciences, the Wenner-Gren Institute, Stockholm University, SE-10691 Stockholm, Sweden. \\ 2 Institute of Molecular Biosciences, University of Graz, A-8010 Graz, Austria. \\ * Corresponding Author: \\ Sabrina Büttner, Department of Molecular Biosciences, the Wenner-Gren Institute, Stockholm University, SE-10691 Stockholm, \\ Sweden; E-mail: sabrina.buettner@su.se
}

\begin{abstract}
The protein phosphatase calcineurin is activated in response to rising intracellular $\mathrm{Ca}^{2+}$ levels and impacts fundamental cellular processes in organisms ranging from yeast to humans. In fungi, calcineurin orchestrates cellular adaptation to diverse environmental challenges and is essential for virulence of pathogenic species. To enable rapid and large-scale assessment of calcineurin activity in living, unperturbed yeast cells, we have generated stable and destabilized GFP transcriptional reporters under the control of a calcineurin-dependent response element (CDRE). Using the reporters, we show that the rapid dynamics of calcineurin activation and deactivation can be followed by flow cytometry and fluorescence microscopy. This system is compatible with live/dead staining that excludes confounding dead cells from the analysis. The reporters provide technology to monitor calcineurin dynamics during stress and ageing and may serve as a drug-screening platform to identify novel antifungal compounds that selectively target calcineurin.
\end{abstract}

doi: $10.15698 / \operatorname{mic} 2020.04 .713$

Received originally: 13.11.2019;

in revised form: 19.01.2020,

Accepted 29.01.2020,

Published 05.02.2020.

Keywords: Calcineurin, calcium signaling, yeast, destabilized GFP, Crz1, reporter, flow cytometry.
Abbreviations:
$\beta$-gal - $\beta$-galactosidase, CDRE - calcineurin-dependent response element, $\mathrm{CN}$ - calcineurin, $\mathrm{CnA}$ - catalytic subunit of $\mathrm{CN}$, $\mathrm{Cn} B$ - regulatory subunit of $\mathrm{CN}$, GFP - green fluorescent protein, $\mathrm{PI}$ - propidium iodide.

\section{INTRODUCTION}

The $\mathrm{Ca}^{2+} /$ calmodulin-dependent serine/threonine phosphatase calcineurin $(\mathrm{CN})$ is a central component of $\mathrm{Ca}^{2+}$ signaling across species. $\mathrm{CN}$ regulates diverse fundamental processes, ranging from cell wall synthesis, stress responses and adaptation in unicellular organisms [1-3] to proliferation, development, immune responses, apoptosis, synaptic plasticity and memory function in metazoans [4-7]. $\mathrm{CN}$ activity across various cell types and organisms requires a tight spatio-temporal regulation. Both excessive and insufficient activity of this phosphatase have been linked to human disease, in particular to diverse neurodegenerative disorders $[8,9]$. Pharmacological calcineurin inhibitors like FK506 (tacrolismus) or cyclosporin A are in clinical use as immunosuppressants, in particular after organ transplantations [5]. As calcineurin signaling has been shown to contribute to the virulence of a variety of fungal pathogens, including species of Aspergillus, Cryptococcus and Candida, $\mathrm{CN}$ inhibitors - alone or in combination with established antifungal drugs - are also being evaluated for their therapeutic potential to combat fungal infections $[10,11]$.

Calcineurin consists of a regulatory $(\mathrm{CnB})$ and a catalytic $(\mathrm{CnA})$ subunit, which in Saccharomyces cerevisiae are encoded by CNB1 and either CNA1 or CNA2, respectively [7]. $\mathrm{CnB}$ harbors four EF-hand motifs, which bind $\mathrm{Ca}^{2+}$ with different affinities and confer structural and functional properties $[12,13]$. CnA comprises a CnB-binding domain, a calmodulin-binding domain and an autoinhibitory domain, which blocks the active site in resting conditions. $\mathrm{Ca}^{2+}-$ dependent binding of calmodulin to the $\mathrm{CnA} / \mathrm{CnB}-\mathrm{Ca}^{2+} \mathrm{di}-$ mer activates the phosphatase complex by displacing the autoinhibitory domain [7]. Across phyla, CN targets share common recognition sites (PxIxIT and LxVP), which determine enzyme-substrate specificity [6]. In yeast, $\mathrm{CN}$-induced changes in gene expression are mainly mediated by the transcription factor Crz1, the analogue of mammalian NFAT, which upon dephosphorylation by $\mathrm{CN}$ drives transcriptional reprogramming and adaptation $[1,3]$. Apart from this transcriptional response, $\mathrm{CN}$ also dephosphorylates various 
A

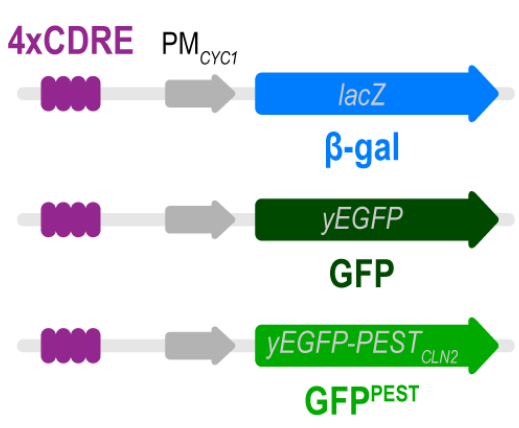

B

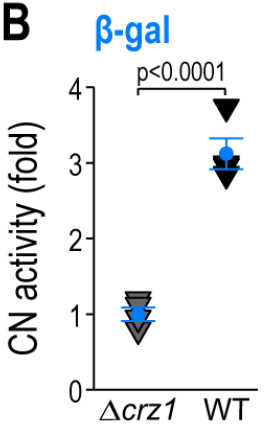

GFP

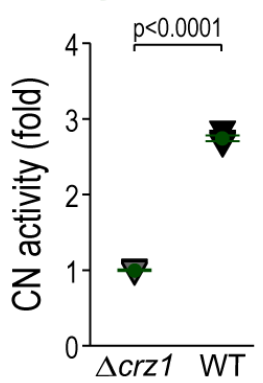

GFPPEST

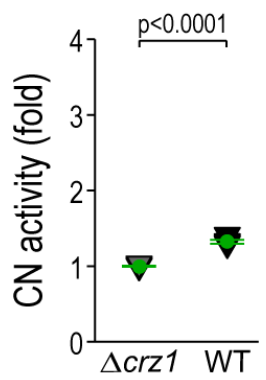

C
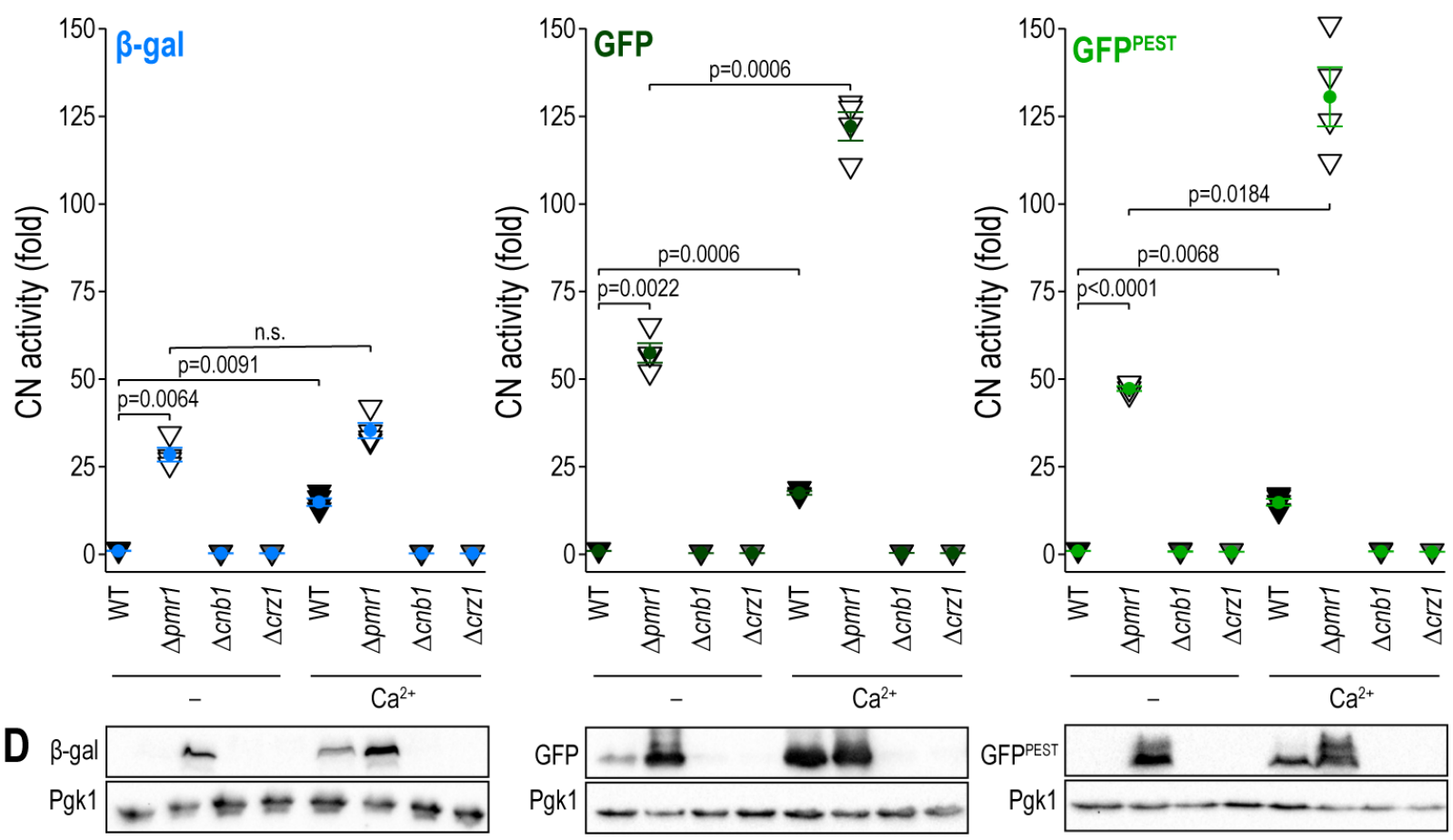

$\mathbf{E}$

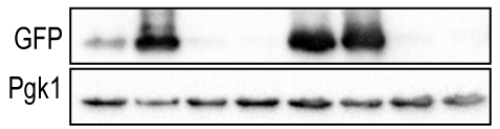

G

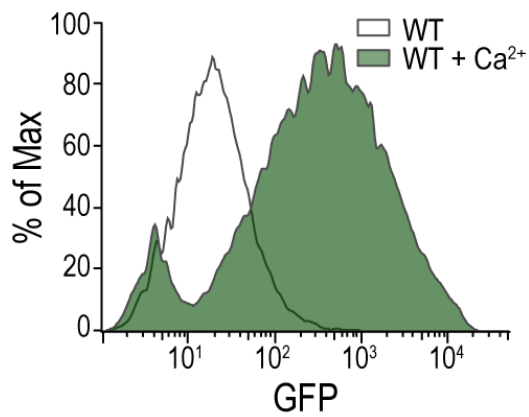

$\mathbf{F}$
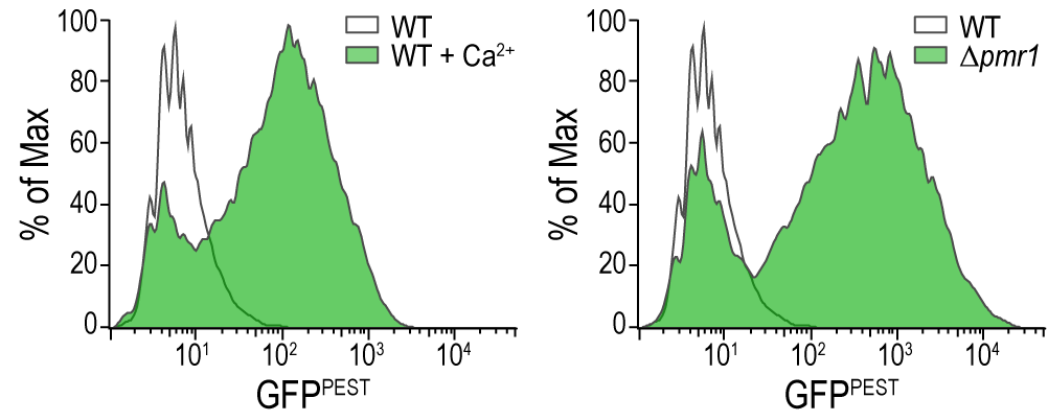

FIGURE 1: GFP and GFPPEST function as reporters of calcineurin activity. (A) Schematics of pAMS366-4XCDRE-lacZ, pAMS366-4xCDRE-GFP and pAMS366-4xCDRE-GFPPEST plasmids encoding reporters for CN activity. (B) CN activity was determined via $\beta$-gal activity or via flow cytometric quantification of GFP fluorescence intensities in exponentially growing wild type and $\triangle c r z 1$ cells equipped with either pAMS3664xCDRE-lacZ, pAMS366-4xCDRE-GFP or pAMS366-4xCDRE-GFPPEST. Values are shown as fold of $\Delta c r z 1$ cells. Means $\pm S E M, n=4$. (C) CN activity was measured as in (B) in exponentially growing wild type, $\Delta p m r 1, \Delta c n b 1$ and $\Delta c r z 1$ cells equipped with the indicated reporter plasmid. For stimulation of $\mathrm{CN}$ activity, cells were treated with $50 \mathrm{mM} \mathrm{Ca}^{2+} 1 \mathrm{~h}$ prior to measurement. Fold of untreated wild type cells is shown. Dead cells were excluded from the analysis via propidium iodide $(\mathrm{PI})$ staining. Means $\pm S E M, n=4$. (D) Representative immunoblots of protein extracts from cells described in (C). Immunoblots were decorated with antibodies against $\beta$-gal or GFP, respectively, and Pgk1 as loading control. (E-G) Histograms of cells quantified in (C) indicating the shift in green fluorescence intensity of wild type cells with and without 50 $\mathrm{mM} \mathrm{Ca}^{2+}$ treatment and $\triangle p m r 1$ cells equipped with the GFP $(\mathrm{E})$ or the GFPPEST reporter $(\mathrm{F}, \mathrm{G})$. 
other substrates, thereby directly impacting processes such as vesicle trafficking, $\mathrm{Ca}^{2+}$ homeostasis, and lipid metabolism [14].

Currently established systems to monitor cellular $\mathrm{CN}$ activity are based on variations of the calcineurindependent response element (CDRE) fused to a reporter gene $[2,14-16]$. Dephosphorylation of $\mathrm{Crz} 1$ by $\mathrm{CN}$ and subsequent binding of $\mathrm{Crz} 1$ to CDREs drives reporter gene expression. The most commonly applied reporters are based on the bacterial $L a c Z$, coding for $\beta$-galactosidase ( $\beta$-gal) [1, $8,12,16,17]$. While the $\beta$-gal assay represents a basic and cost-effective approach, efficiently used to understand diverse aspects of gene expression [18], it comes with clear limitations: it requires a rather lengthy procedure that precludes efficient high-throughput screenings and does not allow for in vivo measurement of $\mathrm{CN}$ activity. Here, we present a reporter system based on GFP that enables rapid and large-scale determination of $\mathrm{CN}$ activity in unperturbed, living cells and allows for a simultaneous discrimination between live and dead cells via flow cytometry. In addition, we utilize a GFP variant fused to a destabilizing tag (degron) to assess $\mathrm{CN}$ activity pulses and magnitudes in higher temporal resolution and demonstrate how dead cell populations confound a proper read-out of $\mathrm{CN}$ activity.

\section{RESULTS AND DISCUSSION}

\section{GFP and GFP PEST reporters capture $\mathrm{CN}$ activation}

To enable large-scale analysis of $\mathrm{CN}$ activity in unperturbed, living yeast cells, we generated reporter constructs coding for a yeast-optimized green fluorescent protein (yEGFP; hereafter referred to as GFP) under the control of a 4-fold repeat of CDRE, allowing Crz1-driven expression of GFP upon dephosphorylation of this $\mathrm{CN}$-responsive transcription factor (Fig. 1A). While the stability of GFP and thus its accumulation over time might be advantageous for instance in scenarios requiring high sensitivity due to marginal $\mathrm{CN}$ activation, it also limits its application as reporter molecule to analyze dynamic and transient changes in gene expression. Thus, we additionally employed GFP destabilized by fusion to a PEST-motif derived from the cyclin Cln2, marking it for rapid proteasomal degradation [23, 25]. We directly compared Crz1/CDRE-driven expression of $\beta$-gal, GFP and GFP PEST to assess CN activity in (i) dividing, unstressed wild type cells, (ii) upon genetic disruption of cellular $\mathrm{Ca}^{2+}$ homeostasis, and (iii) upon administration of high external $\mathrm{Ca}^{2+}$. First, we monitored basal $\mathrm{CN}$ activity in resting conditions in wild type versus $\Delta c r z 1$ cells transformed with the different reporter plasmids. Here, $\Delta c r z 1$ cells served as background, and obtained $\beta$-gal activity values as well as GFP fluorescence intensities are depicted as fold of $\Delta c r z 1$ to allow comparison between the different reporter molecules (Fig. 1B). Determination of $\beta$-gal activity in cell lysates as well as flow cytometric quantification of GFP fluorescence intensity in living cells revealed a $\sim 3$-fold increase of $\mathrm{CN}$ activity compared to $\Delta c r z 1$ cells. A lower $\mathrm{CN}$ activity was detectable in cells expressing the short-lived GFPPEST as reporter, consistent with PEST-driven destabilization and enhanced proteasomal degradation of this molecule (Fig. 1B). To evaluate the different reporter molecules in a context of constitutively active $\mathrm{CN}$, we used cells devoid of Pmr1, an ER/Golgi-localized $\mathrm{Ca}^{2+} / \mathrm{Mn}^{2+}$ ATPase that pumps $\mathrm{Ca}^{2+}$ from the cytosol into lumenal stores. Cells lacking Pmr1 are known to have elevated cytosolic $\mathrm{Ca}^{2+}$ levels and increased CN activity in resting, uninduced conditions [26, 27]. Flow cytometric quantification of fluorescence intensities in vivo in comparison with measurement of $\beta$-gal activity in lysates demonstrated that both GFP and GFP PEST efficiently captured the prominent increase in $\mathrm{CN}$ activity in cells devoid of Pmr1 (Fig. 1C). Cells lacking the regulatory subunit of calcineurin $(\Delta c n b 1)$ as well as $\Delta c r z 1$ cells served as negative controls. To allow direct comparison of the magnitude of $\mathrm{CN}$ activity captured by the three reporter molecules, relative change to wild type was plotted (Fig. 1C). Using immunoblotting as an alternative readout to flow cytometry, we found that similar to $\beta$-gal, the protein levels of GFP and GFP ${ }^{\text {PEST }}$ were markedly increased
A

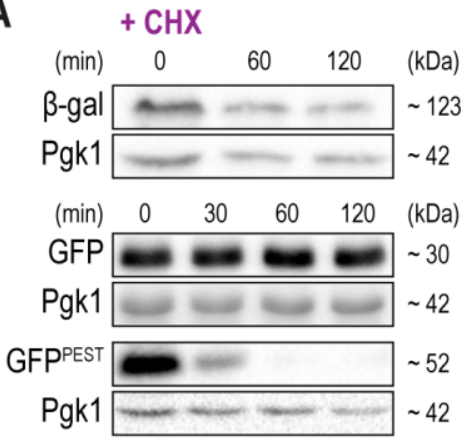

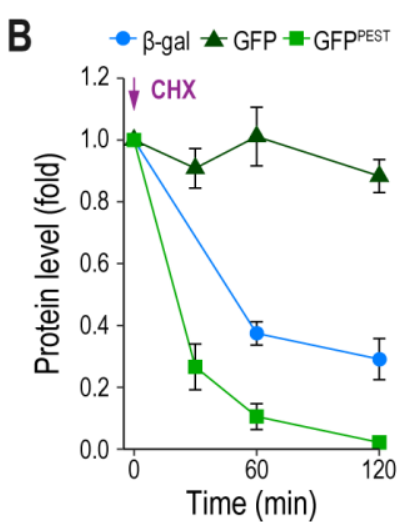

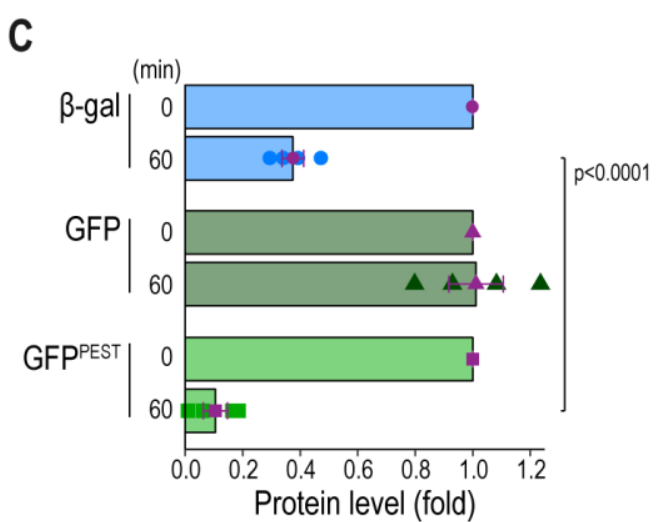

FIGURE 2: Reporter protein stability assessed via immunoblotting. (A-C) Representative immunoblots (A) and corresponding densitometric quantification $(B, C)$ of reporter protein levels in exponentially growing cells lacking Pmr1 equipped with either pAMS366-4xCDRE-lacZ, pAMS366-4xCDRE-GFP or pAMS366-4xCDRE-GFPPEST at indicated times points after addition of cycloheximide (CHX) to a final concentration of $100 \mathrm{mg} / \mathrm{L}$. Blots were decorated with antibodies against $\beta$-galactosidase or GFP, respectively, and Pgk1 as loading control. Molecular weight $(\mathrm{kDa})$ as estimated by Page Ruler Protein standard. Values represent fold to $t=0 \mathrm{~min}$. Means $\pm \mathrm{SEM}, \mathrm{n}=4$. 
in absence of Pmr1 (Fig. 1D). Next, we monitored the response to administration of $50 \mathrm{mM} \mathrm{Ca}^{2+}$, a regime known to activate $\mathrm{CN}$ signaling and nuclear translocation of $\mathrm{Crz} 1$ [15]. Quantification of $\beta$-gal activity and fluorescence intensities revealed a 10 -fold increase of $\mathrm{CN}$ activity in wild type cells $1 \mathrm{~h}$ after $\mathrm{Ca}^{2+}$ administration for all three reporter molecules. Moreover, both GFP as well as GFP ${ }^{\text {PEST }}$ intensities in cells with constitutively high $\mathrm{CN}$ activity ( $\Delta p m r 1)$ further increased upon additional $\mathrm{Ca}^{2+}$ treatment, effectively capturing $\mathrm{CN}$ activities of high amplitudes (Fig. 1C). Corresponding histograms of GFP (Fig. 1E) and GFP PEST (Fig. 1F, G) intensities of untreated versus $\mathrm{Ca}^{2+}$-treated wild type cells as well as of wild type cells versus $\Delta p m r 1$ cells depict the shift in fluorescence intensities as a read-out for $\mathrm{CN}$ activity. In sum, these fluorescence-based systems function as reporters of $\mathrm{CN}$ activity during genetic (permanent) and pharmacological (transiently induced) activation of $\mathrm{CN}$ and are able to effectively capture $\mathrm{CN}$ activities of different magnitudes.

\section{Reporter protein stability assessed via immunoblotting}

Slow turnover of any reporter molecule obstructs the accurate detection of rapid downregulation of gene expression. We monitored protein stability of $\beta$-gal, GFP and GFPPEST after inhibition of de novo protein synthesis with cycloheximide in cells with permanently active $\mathrm{CN}$ due to the absence of Pmr1 (Fig. 2A-C). As expected, immunoblotting demonstrated that GFP was highly stable, and no decay was detectable within 120 min after cycloheximide treatment. While $\beta$-gal protein levels dropped to about $40 \%$ after $60 \mathrm{~min}$, no further degradation was observed over the analyzed time frame, limiting its usefulness as a reporter to detect rapid downregulation of gene expression. In contrast, arrest of translation resulted in a fast reduction of GFPPEST protein levels. $60 \mathrm{~min}$ after cycloheximide addition, about $90 \%$ of GFP PEST was degraded by the pro-

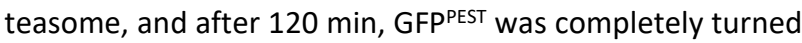
over (Fig. 2A-C). Thus, the proteasomal turnover of GFP PEST is well-suited to capture rapid downregulation of CN activity.

\section{GFP ${ }^{\text {PEST }}$ monitors transient changes in $\mathrm{CN}$ activity}

Next, we tested whether the protein synthesis arrest of stable GFP and destabilized GFPPEST was also readily detectable using fluorescence as a read-out. Therefore, we quantitatively evaluated the fluorescence intensities of the $\mathrm{CN}$ activity reporter molecules via flow cytometry. We treated wild type cells with $50 \mathrm{mM} \mathrm{Ca}^{2+}$ to stimulate $\mathrm{CN}$ activity, which triggered a rapid increase in both GFP and GFPPEST intensities (Fig. 3A, B). Consistent with the protein expression levels, subsequent cycloheximide addition led to a fast decline of GFP PEST but not GFP fluorescence. Corresponding histograms show that both GFP and GFP PEST faithfully reported on the $\mathrm{CN}$ activation upon $\mathrm{Ca}^{2+}$ addition, while only
A $\quad \mathrm{WT}+\mathrm{Ca}^{2+}$

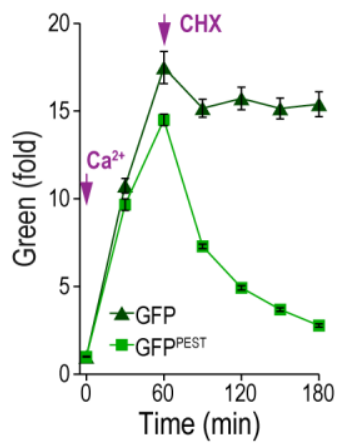

C

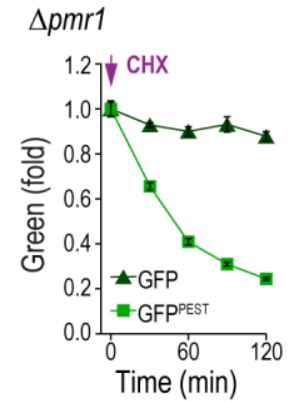

B

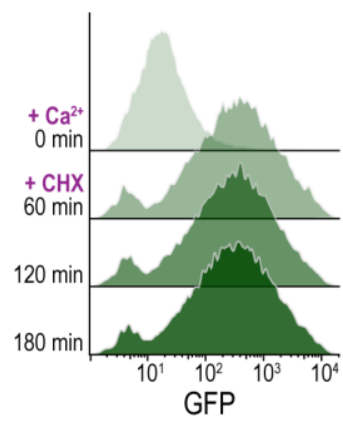

D
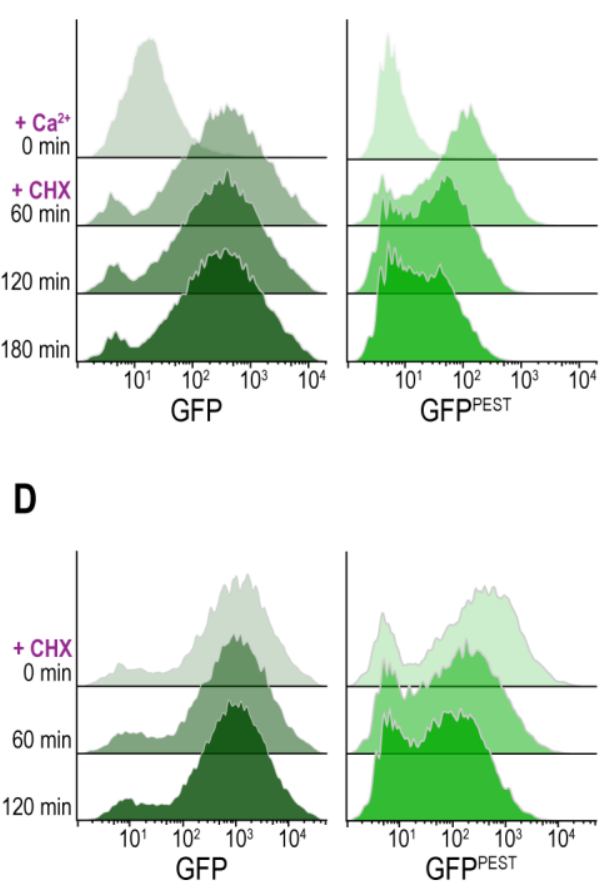

E

G

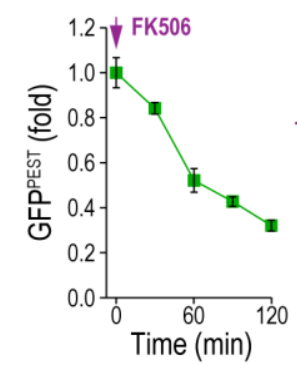

$\mathbf{F}$

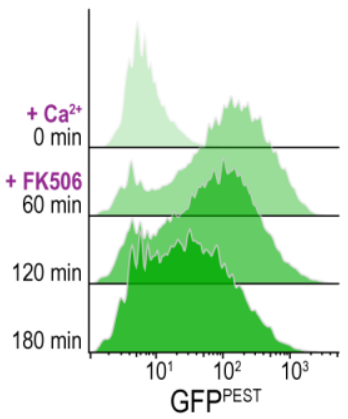

H

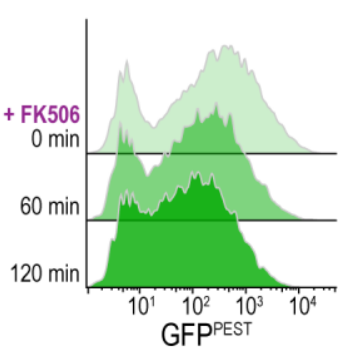

FIGURE 3: GFP PEST as a reporter for transient changes in CN activity. (A-D) Flow cytometric quantification of GFP intensities (A, C) and corresponding histograms of selected time points $(B, D)$ after addition of cycloheximide ( $\mathrm{CHX}$ ) to wild type cells pre-treated with 50 mM Ca ${ }^{2+}$ for 1 $\mathrm{h}(\mathrm{A}, \mathrm{B})$ or to $\Delta p m r 1$ cells $(\mathrm{C}, \mathrm{D})$ equipped with either pAMS366-4xCDRE-GFP or pAMS366-4xCDRE-GFPPEST. (E-H) Flow cytometric quantification of GFP intensities $(E, G)$ and corresponding histograms of selected time points $(F, H)$ after addition of $0.5 \mu M$ FK506 to wild type cells pretreated with $50 \mathrm{mM} \mathrm{Ca}^{2+}$ for $1 \mathrm{~h}(\mathrm{E}, \mathrm{F})$ or to $\Delta p m r 1$ cells $(\mathrm{G}, \mathrm{H})$ equipped with pAMS366-4xCDRE-GFPPEST. Dead cells were excluded from the analysis via propidium iodide $(\mathrm{PI})$ staining. Means $\pm \mathrm{SEM}, \mathrm{n}=4$. 
A
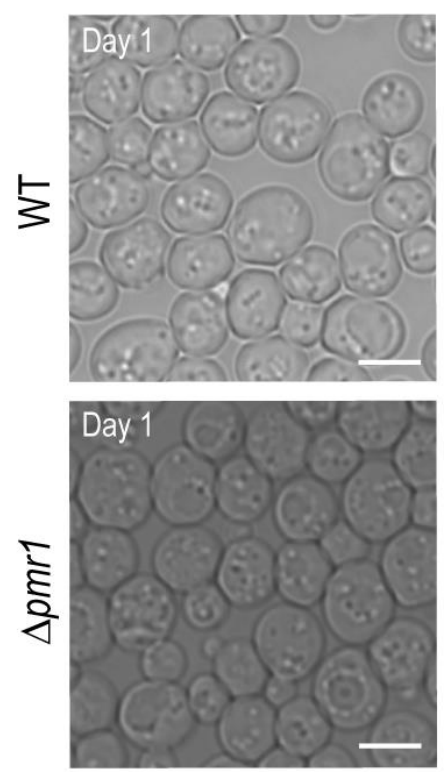

B WT $+\mathrm{Ca}^{2+}$ (day 3$)$

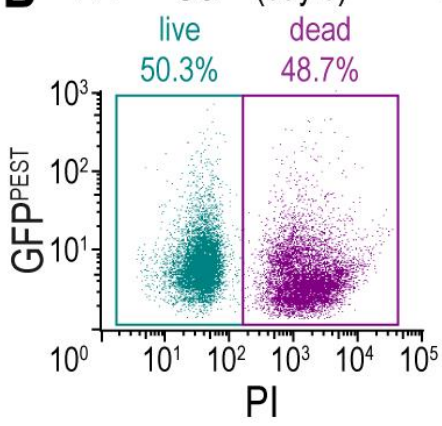

E $\Delta p m r 1$ (day 2)

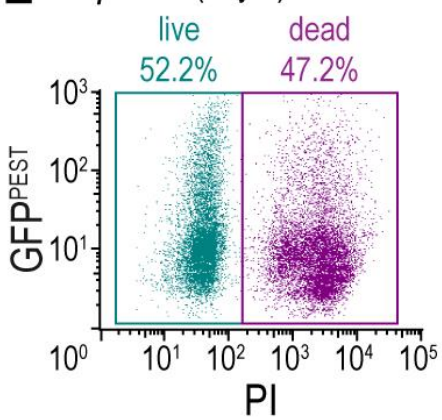

overlay
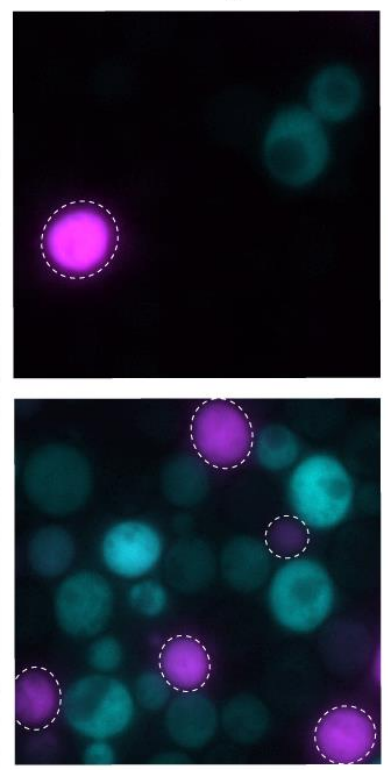

C

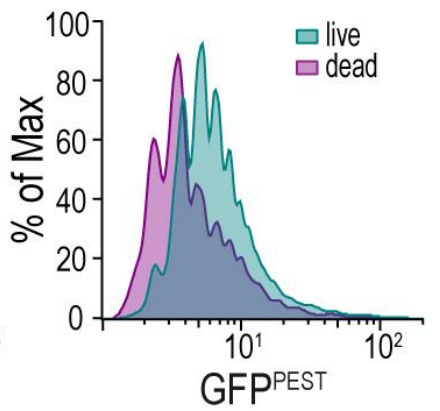

F

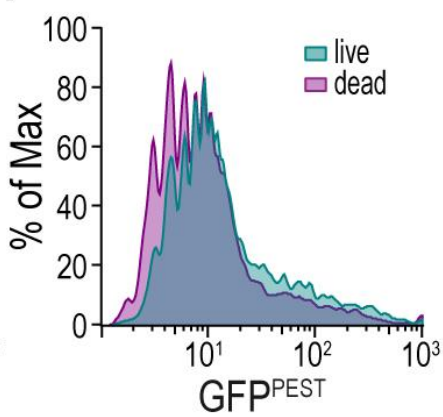

PI
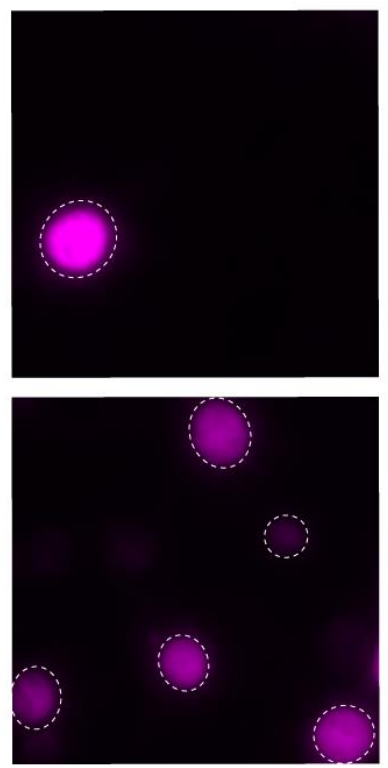

GFPPEST
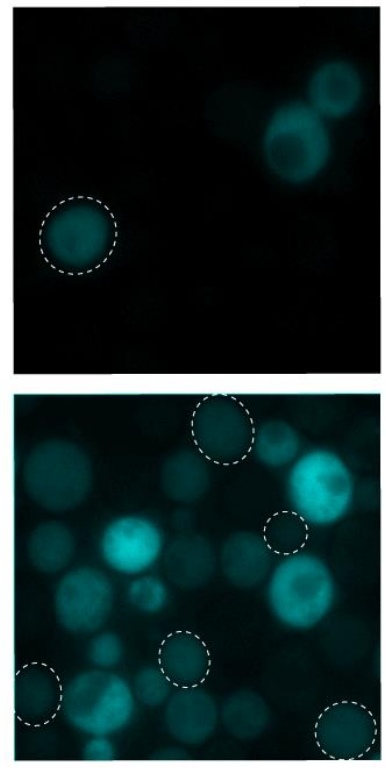

H

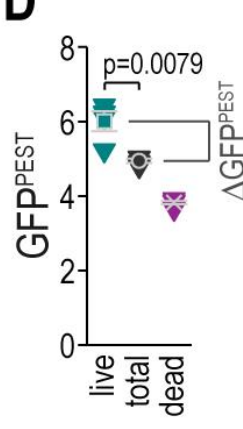

G

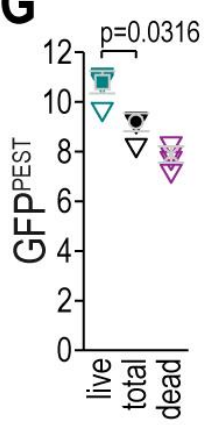

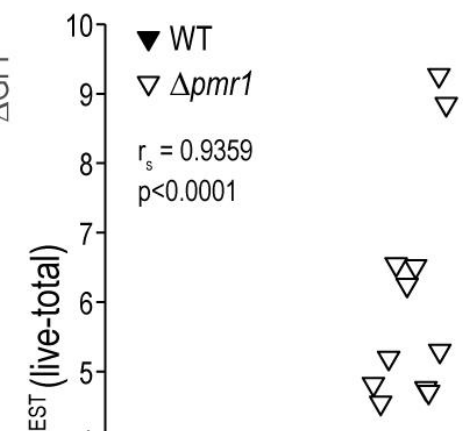

$\nabla$
$\nabla$

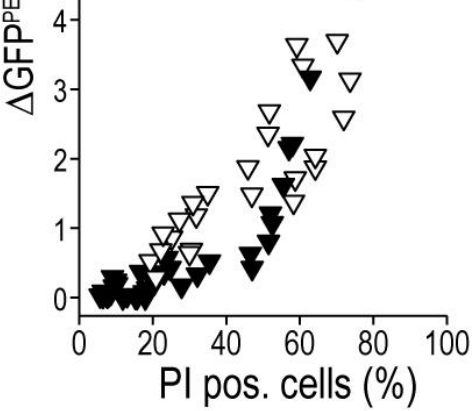

FIGURE 4. A fluorescent reporter for CN activity allows for simultaneous exclusion of dead cells via flow cytometry. (A) Representative micrographs of wild type and $\Delta p m r 1$ cells equipped with pAMS366-4xCDRE-GFPPEST and stained with propidium iodide (PI) to indicate loss of plasma membrane integrity and thus cell death. Scale bar $=5 \mu \mathrm{m}$. (B-G) Flow cytometric analysis of PI-stained aged wild type cells treated with $\mathrm{Ca}^{2+}$ for $1 \mathrm{~h}$ as well as $\triangle p m r 1$ cells equipped with pAMS366-4xCDRE-GFPPEST. Respective dot plots (B, E), histograms of GFPPEST intensities of PI negative (live) and PI positive (dead) cells (C, F) and mean GFPPEST intensities of total, live and dead cell populations (D, G) are shown. (H) Simultaneous quantification of cell death via PI staining and CN activity in cells equipped with pAMS366-4xCDRE-GFPPEST. Wild type and $\triangle p m r 1$ cells of different age and upon treatment with different concentrations of $\mathrm{Ca}^{2+}$ were analyzed, and the difference of GFPPEST fluorescence intensity between total and live cell populations ( $\triangle$ GFPPEST (live-total)) was plotted against the percentage of PI positive cells. 
GFPPEST captured the shut down upon cycloheximide treatment (Fig. 3A, B). In this line, stop of translation in $\Delta p m r 1$ cells, which exhibit permanently high $\mathrm{CN}$ activity, triggered a rapid decline of GFPPEST fluorescence, while GFP intensities were mainly unaffected (Fig. 3C, D). Ultimately, we confirmed that GFP PEST captures also a decline of $\mathrm{CN}$ activity upon pharmacological inhibition of $\mathrm{CN}$. To this end, we used FK506, which in complex with the immunophilin FKBP12 specifically inhibits CN across species [7, 11]. Indeed, addition of FK506 to $\mathrm{Ca}^{2+}$-treated wild type cells (Fig. $3 \mathrm{E}, \mathrm{F}$ ) as well as to $\Delta p m r 1$ cells with high resting $\mathrm{CN}$ activity (Fig. 3G, H) led to a rapid drop in CN activity as assessed by flow cytometric quantification of GFP PEST intensities. In sum, both reporters permit in vivo monitoring of $\mathrm{CN}$ activity and recapitulate the rapid stimulation of this phosphatase. While GFP PEST additionally enables the assessment of CN deactivation due to its fast proteasomal turnover, the accumulation of the more stable GFP can serve to monitor marginal $\mathrm{CN}$ activities close to background.

\section{Flow cytometric analysis enables simultaneous exclusion of dead cells}

Dead or dying cells in a culture confound the result of any readout if not accounted for, especially as those cells and debris thereof contribute to the optical density that frequently is used for normalization. The use of fluorescent reporters combined with a live/dead staining allows for the simultaneous analysis of $\mathrm{CN}$ activity and cell death. We employed propidium iodide (PI), indicative of loss of plasma membrane integrity, to discriminate between live and dead cell populations (Fig.4A-G). As expected, flow cytometric evaluation and appropriate gating of the distinct subpopulations demonstrated that simply assessing CN activity in the total cell population leads to a significant underestimation of the actual $\mathrm{CN}$ activity of living cells (Fig. 4B-G). To evaluate the impact of dead cell populations on actual $\mathrm{CN}$ activity, we simultaneously monitored cell death and $\mathrm{CN}$ activity in wild type and $\Delta p m r 1$ cultures of different cellular age and upon treatment with various concentrations of $\mathrm{Ca}^{2+}$. For each condition, we determined the shift in GFP fluorescence intensity upon exclusion of the dead cell population ( $\triangle$ GFP PEST) (Fig. $4 \mathrm{H}$ ). Plotting $\triangle$ GFPPEST against the percentage of cell death determined via $\mathrm{PI}$ staining shows a clear positive correlation (Spearman correlation coefficient $r_{s}=0.9359, p<0.001$, Fig. $4 \mathbf{H}$ ). Thus, combining a fluorescent reporter molecule with a live/dead staining facilitates accurate assessment of $\mathrm{CN}$ activity in cultures with larger populations of dead cells, for instance upon ageing, genetic modification or drug treatment. In addition, a flow cytometric read-out of fluorescence will also enable a direct correlation between the degree of $\mathrm{CN}$ activity in each cell and any other parameter using appropriate fluorescent dyes or tags.

Collectively, our results demonstrate that CDRE-driven expression of GFP and GFP PEST serves as effective reporter to monitor $\mathrm{CN}$ activity via flow cytometry, fluorescence microscopy or immunoblotting. Use of the short-lived GFPPEST enables the assessment of rapid and dynamic changes in $\mathrm{CN}$ activity, whereas the stability of GFP render it suitable to report on more subtle changes in base-line activities of the phosphatase in unstressed, resting cells. In combination with a live/dead staining, our system represents an efficient screening platform to simultaneously evaluate compounds for $\mathrm{CN}$ inhibitory activity and toxicity.

\section{MATERIALS AND METHODS}

\section{Yeast strains and culture conditions}

$S$. cerevisiae strains used in this study were all derived from BY4741 (MATa; his $3 \Delta 1$; leu2 $\Delta 0$, met15 $\Delta 0$; ura $3 \Delta 0$, Euroscarf) CRZ1 (BY4741 crz1::hphNT1) and CNB1 (BY4741 cnb1::hphNT1) deletion mutants have been described previously [19]. The PMR1 deletion mutant was generated via homologous recombination following established protocols [20]. Oligonucleotides 5'-CAG CAC AGA CGT AAG CTT AAG TGT AAG TAA AAG ATA AGA TAA TCA GCT GAA GCT TCG TAC GC-3' and 5'-TAA CAG AGA CAG TCC AAC GGC GTA GTT GAA CAT TTT GTT GCA TAG GCC ACT AGT GGA TCT G-3' used to amplify the gene disruption cassette from pUG6 were designed to leave HUR1 intact, which overlaps with the C-terminal region of $P M R 1$ on the complementary strand (BY4741 pmr1::kanMX4 HUR1 intact). Plasmid transformation was performed as previously described [21]. Cells were grown in synthetic complete medium containing $0.17 \%$ yeast nitrogen base (Difco, BD Biosciences), $0.5 \%\left(\mathrm{NH}_{4}\right)_{2} \mathrm{SO}_{4}, 2 \%$ glucose, $30 \mathrm{mg} / \mathrm{l}$ adenine and $30 \mathrm{mg} / \mathrm{l}$ of all amino acids (except for $80 \mathrm{mg} / \mathrm{l}$ histidine and $200 \mathrm{mg} / \mathrm{l}$ leucine) and lacking uracil for reporter plasmid selection. All components were prepared separately as stocks and mixed after autoclaving $\left(20 \mathrm{~min}, 121^{\circ} \mathrm{C}, 210 \mathrm{kPa}\right)$ : $10 \mathrm{x}$ YNB with $\left(\mathrm{NH}_{4}\right)_{2} \mathrm{SO}_{4}, 10 \mathrm{x}$ amino acids + adenine mixture, $20 \%$ glucose. Cells were treated with $50 \mathrm{mM} \mathrm{CaCl}_{2}$ (stock $2 \mathrm{mM} \mathrm{CaCl}_{2}$ ), $100 \mathrm{mg} / \mathrm{L}$ cycloheximide (stock $10 \mathrm{mg} / \mathrm{ml}$; Sigma) at indicated time points. Overnight cultures incubated for $16-20 \mathrm{~h}$ where used to inoculate cultures to OD 0.1. For standard experiments (cell death analysis, fluorescence intensity measurements and epifluorescence microscopy) cells were cultivated in 96-deep well plates at $28^{\circ} \mathrm{C}, 999 \mathrm{rpm}$. For experiments requiring larger amounts of cells ( $\beta$-gal assay and immunoblot analysis), cells were cultivated in $125 \mathrm{ml}$ baffled Erlenmeyer flasks with cellulose stoppers at $28^{\circ} \mathrm{C}, 145 \mathrm{rpm}$. To rule out clonogenic variation, all experiments were performed with four different transformants.

\section{Construction of fluorescence-based reporter plasmids}

The reporter constructs generated and used in this study are illustrated in Figure $1 \mathrm{~A}$ and have been deposited at Addgene (IDs: 138657 and 138658), including plasmid maps and sequencing data. Table 1 summarizes the most relevant features of the constructs. For the GFP version (pAMS366-4xCDRE-GFP), yEGFP was amplified from PYM25 [22] with primers $5^{\prime}$-ATC TGG ATC CAT GTC TAA AGG TGA AGA ATT ATT CAC-3' and 5'ATC TGA GCT CTT ATT TGT ACA ATT CAT CCA TAC C- 3 ' and ligated into pAMS366-4xCDRE (kind gift from M. Cyert) [1], cut with restriction enzymes BamHI and Sacl (Thermo Scientific FastDigest). For the GFPPEST version (pAMS366-4xCDREGFPPEST), pAMS366-4xCDRE was cut with BamHI (Thermo Scientific FastDigest) and ligated with yEGFP3-PEST CLN2 amplified from pSVA13 (kind gift from S. Avery) [23] with primers 5'-ATC TGG ATC CAT GTC TAA AGG TGA AGA ATT ATT CAC- $3^{\prime}$ and $5^{\prime}$ ATC TGG ATC CCT ATA TTA CTT GGG TAT TGC CCA TAC C-3'. 
TABLE 1. Specifications for PAMS366-4xCDRE-GFP and PAMS366-4xCDRE-GFPPEST.

\begin{tabular}{lcc}
\hline & 4xCDRE-GFP & 4xCDRE-GFPPEST \\
\hline Vector backbone & pAMS366 [1] & pAMS366 [1] \\
\hline Copy number & high $(2 \mu)$ & high $(2 \mu)$ \\
\hline Reporter & yeGFP [22] & yEGFP3-PEST CLN2 [23] \\
\hline Reporter half-life & $>120$ min & $<30$ min \\
\hline $\begin{array}{l}\text { Auxotrophic } \\
\text { marker }\end{array}$ & URA3 & URA3 \\
\hline $\begin{array}{l}\text { Antibiotic } \\
\text { resistance }\end{array}$ & Amp & Amp \\
\hline
\end{tabular}

Proper insertion of the PCR product into the vector backbone was confirmed by sequencing (Eurofins) with primers 5'-GTG GGT TTA GAT GAC AAG GGA GAC G-3' and 5'-CGT ACT GTG AGC CAG AGT TG-3' (for the GFP variant) and primers 5'-GTG GGT TTA GAT GAC AAG GGA GAC G-3', 5'-CAC AAA TTT TCT GTC TCC G-3' and 5'-GTC TTG TTA CCA GAC AAC C-3' (for the GFPPEST variant).

\section{$\beta$-gal assay}

$\beta$-gal assay was essentially performed as described in [24]. In brief, $1 \mathrm{OD}$ of cells was harvested, washed and resuspended in $250 \mu \mathrm{l}$ Z-buffer $\left(60 \mathrm{mM} \quad \mathrm{Na}_{2} \mathrm{HPO}_{4} * 7 \quad \mathrm{H}_{2} \mathrm{O}, 40 \mathrm{mM}\right.$ $\mathrm{Na}_{2} \mathrm{H}_{2} \mathrm{PO}_{4}{ }^{*} \mathrm{H}_{2} \mathrm{O}, 10 \mathrm{mM} \mathrm{KCl}, 1 \mathrm{mM} \mathrm{MgSO}$, adjusted to $\mathrm{pH} 7.0$ and $40 \mathrm{mM}$ 2-mercaptoethanol). An aliquot of this suspension was used to measure $\mathrm{OD}_{600}$ for normalization. The remaining cells were lysed by addition of $0.01 \%$ SDS and $10 \%$ chloroform (final concentrations) and vortexing. To start and stop the enzymatic reaction, $0.36 \mathrm{mg} / \mathrm{ml}$ ONPG in Z-buffer and $0.2 \mathrm{M}$ $\mathrm{Na}_{2} \mathrm{CO}_{3}$ (final concentrations) were added, respectively. After centrifugation ( $400 \mathrm{~g}, 5 \mathrm{~min}, 21^{\circ} \mathrm{C}$ ), an aliquot of the suspension was taken to measure $\mathrm{OD}_{405}$ at a 2300 EnSpire Multimode Plate Reader (PerkinElmer) equipped with an excitation double monochromator (application: well area scan, averaging 6 measurement repeats). $\beta$-gal activity was calculated as (1000 $\mathrm{x}$ $\mathrm{OD}_{405} \times$ dilution factor $) /\left(\mathrm{OD}_{600} \mathrm{x}\right.$ reaction time $\mathrm{x}$ volume in reaction). Per genotype and condition, four isogenic mutants were analyzed.

\section{Immunoblot analysis}

6 OD of cells were harvested and lysed with $250 \mu \mathrm{l}$ buffer containing $1.85 \mathrm{M} \mathrm{NaOH}$ and $7.5 \% \beta$-mercaptoethanol. The suspension was incubated at $4^{\circ} \mathrm{C}$ for $10 \mathrm{~min}, 250 \mu \mathrm{l} 55 \%$ trichloroacetic acid were added and incubated again at $4^{\circ} \mathrm{C}$ for 10 min. After centrifugation $\left(4^{\circ} \mathrm{C}, 10 \mathrm{~min}, 10000 \mathrm{~g}\right)$ trichloroacetic acid was removed and pellets were resuspended in Urea Loading Buffer (200 mM Tris/ $\mathrm{HCl}, 8 \mathrm{M}$ urea, 5\% SDS, $1 \mathrm{mM}$ EDTA, 0.02\% bromophenol blue, $15 \mathrm{mM}$ DTT, pH 6.8). SDSPAGE with Tris-glycine running buffer ( $250 \mathrm{mM}$ Tris base, 0.2 M glycine, $0.05 \%$ SDS (w/v)) was performed in a SE250 Mighty Small II Mini Vertical Protein Electrophoresis Unit (Hoefer) according to manufacturer's settings. For wet protein transfer onto a PVDF membrane (T830.1, Roth) with CAPS buffer (10 mM CAPS, 10\% EtOH, pH 11) a TE22 Mighty Small Transfer
Tank (Hoefer) was used. Blocking was performed with $5 \%$ milk powder in Tris buffered saline $(50 \mathrm{mM}$ Tris, $150 \mathrm{mM} \mathrm{NaCl}, \mathrm{pH}$ 7.4). For immunoblotting anti-GFP (1:2500, 11814460001 Roche), anti-Pgk1 (1:10000, 459250, Invitrogen) and a peroxidase-conjugated secondary anti-mouse antibody (1:10000, A9044, Sigma-Aldrich) were used. Chemiluminescence imaging was performed on a ChemiDoc ${ }^{\mathrm{TM}}$ XRS+ (BioRad) with ImageLab software ( $v$ 5.2.1) and the application Chemi Hi Resolution (no illumination, no filter, $2 \times 2$ binning, manual exposure times). The same software was used for densitometric quantification.

\section{Cell death analysis, epifluorescence microscopy and flow cytometry}

PI staining as an indicator of loss of membrane integrity was used to assess cell death and was essentially performed as previously described [19]. In brief, approximately $1 \times 10^{6}$ cells per sample were harvested in 96-well plates and incubated for $5 \mathrm{~min}$ with PI (81845, Sigma), final concentration $500 \mathrm{ng} / \mathrm{ml}$ in phosphate buffered saline $(25 \mathrm{mM}$ potassium phosphate, $0.9 \% \mathrm{NaCl}$; adjusted to $\mathrm{pH} 7.2$ ). Micrographs were recorded using a ZEISS Axioplan2 microscope, (63x/1.40 oil objective, Cy5 (propidium iodide) and FITC (GFP) filters) with ZEISS Axio cam MRm camera and AxioVision40x64 software (v4.9.1.0). Flow cytometry was performed on a Guava easyCyte 5HT equipped with a $50 \mathrm{~mW} 488 \mathrm{~nm}$ laser (blue) and the following filters: 488/16 (SSC), 525/30 (green), 695/50 (red) (Merck group). Per genotype and condition, four isogenic mutants were analyzed. All samples were measured in the staining solution and each sample was resuspended automatically by mixing for 5 seconds before acquisition. Per sample, 5000 events were recorded (threshold parameter FSC-HLog, value 100). Data was acquired and analyzed with InCyte software (3.1).

\section{Data preparation and statistical analysis}

Data were analyzed and graphs were generated with $R$ version 3.5.1 (base, ggplot2 and dplyr packages) or FlowJo 10.6.1 (histograms and dot plots Fig. 4B, E). Figures were prepared in Adobe Illustrator CC 2017. Data are presented as dot plots or line graphs and, where applicable, with means and error bars showing standard error of mean (SEM). Histograms contain concatenated data of all transformants analyzed. Y-axis in all histograms is \% of Max, where the mode in every population is scaled to $100 \%$. For all data sets, normal distribution (ShapiroWilk's) and homogeneity of variances (Levene's) was confirmed (OriginPro 2017 b9.2.4.380) (except data depicted in Fig. $4 \mathrm{H}$, please see below). To compare reporter protein levels (between-subject) over time (within-subject), a two-way ANOVA mixed design was performed with Tukey post hoc test for one variable (protein levels, Fig. 2C). For comparisons between two groups, an unpaired two-way Student's t-test was performed (Fig. 1B). For comparison of fold changes in Figure $1 C$, all conditions were treated as individual groups (independent variable) and analyzed with Brown-Foresythe and Welch ANOVA with Dunnett's T3 multiple comparison (Prism GraphPad v8.2.1) as there was no homogeneity of variances. Normality was given for all groups except for untreated $\Delta c n b 1$ cells equipped with GFPPEST, which was ignored due to equal sample size in all groups. Cell death and $\triangle$ GFPPEST were correlated with two-tailed Spearman rank test (nonparametric, as 
data was not normally distributed, Fig. $4 \mathrm{H})$. P values are stated with 4 decimals or as n.s. (not significant, $p>0.05$ ).

\section{ACKNOWLEDGMENTS}

This work was supported by the Swedish Research Council, the Austrian Science Fund FWF (P27183-B24), Knut and Alice Wallenberg Foundation and Olle Engkvists Stiftelse.

\section{CONFLICT OF INTEREST}

The authors declare no conflict of interest.

\section{COPYRIGHT}

(C) 2020 Diessl et al. This is an open-access article released under the terms of the Creative Commons Attribution (CC BY) license, which allows the unrestricted use, distribution, and reproduction in any medium, provided the original author and source are acknowledged.

Please cite this article as: Jutta Diessl, Arpita Nandy, Christina Schug, Lukas Habernig and Sabrina Büttner (2020). Stable and destabilized GFP reporters to monitor calcineurin activity in Saccharomyces cerevisiae. Microbial Cell 7(4): 106-114. doi: 10.15698/mic2020.04.713

12. Connolly S, Quasi-Woode D, Waldron L, Eberly C, Waters K, Muller EM, and Kingsbury TJ (2018). Calcineurin Regulatory Subunit CalciumBinding Domains Differentially Contribute to Calcineurin Signaling in Saccharomyces cerevisiae. Genetics genetics.300911.2018. doi: 10.1534/genetics.118.300911

13. Feng B, and Stemmer PM (1999). Interactions of Calcineurin A, Calcineurin B, and Ca2+. Biochemistry 38(38): 12481-12489. doi: 10.1021/bi990492w

14. Goldman A, Roy J, Bodenmiller B, Wanka S, Landry CR, Aebersold $R$, and Cyert MS (2014). The calcineurin signaling network evolves via conserved kinase-phosphatase modules that transcend substrate identity. Mol Cell 55(3): 422-435. doi: 10.1016/j.molcel.2014.05.012

15. Cai L, Dalal CK, and Elowitz MB (2008). Frequency-modulated nuclear localization bursts coordinate gene regulation. Nature 455(7212): 485-490. doi: 10.1038/nature07292

16. Araki $Y$, Wu H, Kitagaki $H$, Akao T, Takagi $H$, and Shimoi $H$ (2009). Ethanol stress stimulates the $\mathrm{Ca} 2+-$ mediated calcineurin/ $\mathrm{Crz1}$ pathway in Saccharomyces cerevisiae. J Biosci Bioeng 107(1): 1-6. doi: 10.1016/j.jbiosc.2008.09.005

17. Niedzwiecka K, Tisi R, Penna S, Lichocka M, Plochocka D, and Kucharczyk $R$ (2018). Two mutations in mitochondrial ATP6 gene of ATP synthase, related to human cancer, affect ROS, calcium homeostasis and mitochondrial permeability transition in yeast. Biochim Biophys Acta 1865(1): 117-131. doi: 10.1016/j.bbamcr.2017.10.003

18. Juers DH, Matthews BW, and Huber RE (2012). LacZ $\beta$ galactosidase: structure and function of an enzyme of historical and molecular biological importance. Protein Sci 21(12): 1792-1807. doi: 10.1002/pro.2165

19. Aufschnaiter A, Habernig L, Kohler V, Diessl J, Carmona-Gutierrez $D$, Eisenberg T, Keller W, and Büttner S (2017). The Coordinated Action of Calcineurin and Cathepsin D Protects Against $\alpha$-Synuclein Toxicity. Front Mol Neurosci 10: 207. doi: 10.3389/fnmol.2017.00207

20. Güldener U, Heck S, Fielder T, Beinhauer J, and Hegemann JH (1996). A new efficient gene disruption cassette for repeated use in budding yeast. Nucleic Acids Res 24(13): 2519-2524. doi 10.1093/nar/24.13.2519

21. Gietz RD, and Woods RA (2006). Yeast transformation by the LiAc/SS Carrier DNA/PEG method. Methods Mol Biol 313: 107-120. doi: 10.1385/1-59259-958-3:107

22. Janke C, Magiera MM, Rathfelder N, Taxis C, Reber S, Maekawa $H$, Moreno-Borchart A, Doenges G, Schwob E, Schiebel E, and Knop M (2004). A versatile toolbox for PCR-based tagging of yeast genes: new fluorescent proteins, more markers and promoter substitution cassettes. Yeast 21(11): 947-962. doi: 10.1002/yea.1142 
23. Mateus C, and Avery SV (2000). Destabilized green fluorescent protein for monitoring dynamic changes in yeast gene expression with flow cytometry. Yeast 16(14): 1313-1323. doi: 10.1002/10970061(200010)16:14<1313::AID-YEA626>3.0.CO;2-O

24. Griffith $\mathrm{KL}$, and Wolf RE (2002). Measuring beta-galactosidase activity in bacteria: cell growth, permeabilization, and enzyme assays in 96-well arrays. Biochem Biophys Res Commun 290(1): 397-402. doi: $10.1006 /$ bbrc. 2001.6152

25. Salama SR, Hendricks KB, and Thorner J (1994). G1 cyclin degradation: the PEST motif of yeast Cln2 is necessary, but not sufficient, for rapid protein turnover. Mol Cell Biol 14(12): 7953-7966. doi: 10.1128/mcb.14.12.7953
26. Zhao Y, Yan H, Happeck R, Peiter-Volk T, Xu H, Zhang Y, Peiter E, van Oostende Triplet $C$, Whiteway $M$, and Jiang $L$ (2016). The plasma membrane protein Rch1 is a negative regulator of cytosolic calcium homeostasis and positively regulated by the calcium/calcineurin signaling pathway in budding yeast. Eur J Cell Biol 95(3-5): 164-174. doi: 10.1016/j.ejcb.2016.01.001

27. Locke EG, Bonilla M, Liang L, Takita Y, and Cunningham KW (2000). A homolog of voltage-gated $\mathrm{Ca}(2+)$ channels stimulated by depletion of secretory $\mathrm{Ca}(2+)$ in yeast. Mol Cell Biol 20(18): 6686-6694. doi: $10.1128 / \mathrm{mcb} .20 .18 .6686-6694.2000$ 